\title{
Uncertainty in vision based modal analysis: probabilistic studies and experimental validation
}

\author{
Alberto Lavatelli, Emanuele Zappa \\ Department of Mechanical Engineering, Politecnico di Milano, Via La Masa 120156 Milano
}

\begin{abstract}
This paper analyses the issues related with the application of photogrammetric methods in the context of modal analysis. In this field of mechanical testing, the vibrations of the measurand are monitored. The possibility to measure displacement of several monitored points without directly instrumenting those, pushes the interest toward the application of an image based measurement system. However, the process of acquiring a moving target with a camera is complex due to the motion blur phenomenon. The effects of this particular phenomenon on uncertainty generation and propagation are discussed in parallel with Monte Carlo simulation and experimental approach. The case study here proposed focuses on the estimation of the main modal parameters (first two resonant frequencies and amplitudes) of a structure with the help of 2D Blob Analysis, with particular focus in comparing the performances of photogrammetry with the ones of classic motion transducers. The results of both the approaches confirm that the choice of using photogrammetry as an input for modal analysis should be made carefully, since the actual behavior of a structure can be distorted. Nonetheless, the results show that particular care should be made in the choice of structural excitation when using vision based techniques.
\end{abstract}

\section{Section: RESEARCH PAPER}

Keywords: uncertainty analysis; photogrammetry; modal analysis; vision systems; vibrations;

Citation: Alberto Lavatelli, Emanuele Zappa, Uncertainty in vision based modal analysis: probabilistic studies and experimental validation, Acta IMEKO, vol. 5 , no. 4, article 7, December 2016, identifier: IMEKO-ACTA-05 (2016)-04-07

Section Editor: Lorenzo Ciani, University of Florence, Italy

Received October 3, 2016; In final form November 22, 2016; Published December 2016

Copyright: () 2016 IMEKO. This is an open-access article distributed under the terms of the Creative Commons Attribution 3.0 License, which permits unrestricted use, distribution, and reproduction in any medium, provided the original author and source are credited

Corresponding author: Alberto Lavatelli, e-mail: alberto.lavatelli@polimi.it

\section{INTRODUCTION}

In the relevant field of structural health monitoring (SHM) it is possible to highlight several possible applications of vision based monitoring techniques. In particular, the possibility to measure the displacement of several monitored points without directly instrumenting those pushes the interest toward the application of image based vibration measurement systems.

Even if vision based SHM systems have been proposed [1][5], the application of those techniques is still limited. One main reason is the difficulty found in the management of uncertainty. In fact, the process of image formation when dealing with moving targets, variable lighting and optical non-linearity is hardly predictable. In some circumstances (such as indoor tests of highly regular mechanical systems) it may be possible to describe the whole image formation process, so that all the parameters of the optical transfer function are known. Nonetheless, the effect of motion blur, lighting variations and optical aberration on the final measurement accuracy is different with respect to each possible image analysis technique. Therefore, also the uncertainty connected to the extraction of metrological information from a set of images is hard to predict.

Furthermore, the dynamic behaviour of actual structures is itself uncertain: given a certain input, the frequency response in terms of displacement and acceleration is variable by stochastic means [6]-[9].

Given those premises, the aim of this paper is to find a robust solution to approach the problem of uncertainty estimation when monitoring a vibrating mechanical system with a vision based measurement system. Due to complexity of the system under analysis the solution sees the application of a probabilistic framework. This choice follows the recommendation of the ISO GUM standard [10], where a detailed chapter is dedicated to the analysis of uncertainty by 
means of Monte Carlo Techniques [11]. The solution found is then tested with an experiment designed on purpose.

Eventually, this paper proposes both a probabilistic and experimental analysis of uncertainty. On one hand, this choice is justified by the interest in giving an experimental confirmation of the findings proposed by the Monte Carlo simulation. On the other hand, this procedure tries to provide a more complete view on the uncertainty analysis problem. In fact, despite any possible partial knowledge of uncertainty sources, the process of uncertainty propagation and estimation may itself be under discussion, since it relies on the model chosen to represent uncertainty itself. From a statistical point of view, uncertainty can be derived from a Bayesian or frequentist approach. Monte Carlo methods in particular, belongs to the first family, since the results returned are conditional probabilities [12]. The experimental approaches, conversely, are hard to be said as Bayesian, since the observation of the phenomenon is mediated by an instrument who has its own stochastic behaviour. Furthermore, the occurrences of measurements are computed upon the recorded output of a sensor. Fortunately, for what concerns most of the engineering testing (such as in most wind-tunnel tests, for example) it is possible to say that the choice between a Bayesian or frequentist model will not introduce large variation in the significance of the numbers computed using them [13]. Nonetheless, from a theoretical point of view, several recent works propose a substantial unification of the two approaches [14]-[16]. In this sense, the contemporary presence of a Monte Carlo analysis and an experimental analysis of uncertainty accords with the previous unification statement.

\section{CAMERA BASED MEASUREMENT OPERATING PRINCIPLE}

The practice of extracting metrological information from an acquired image is well established through the years and it is generally named as photogrammetry. More in details, photogrammetry is a contact-less measurement approach that uses a series of recorded images to identify coordinates of points, patterns, and features in the images or to detect the boundaries of objects [17]. A series of images enables the operation of tracking the motion of a given measurand, hence vibration monitoring can be carried out by means of vision based techniques. A single camera is able to measure a planar motion or 3D motion of known points on rigid bodies [18], [19], while camera pairs are necessary to monitor 3D motion of generic objects.

Without loss of generality, photogrammetry is performed on digital images. The formation of a digital image converts the appearance of a 3D object into a discretized 2D planar projection of the same object. This procedure involves a cascade of optoelectronic phenomena that is known as the image formation process. Although complex, image formation has been modelled successfully by several scientists [20], [21]. In the context of computer vision application for mechanical measurements, the most widely accepted solution is the one proposed by Zhang [22], who proposed also an efficient way to calibrate cameras and recover the image formation model parameters. As for every calibration procedure, uncertainty exists and involves several aspects of photogrammetry. Considering static images, the main sources of uncertainty are due to optical aberration, image blur, non-uniform luminance and limited image resolution, whose presence generates uncertainty in the estimation of the intrinsic and extrinsic parameters of a camera system. The fore mentioned sources do not appear on calibration only, since they appear also on the generic image of the measurand. When recording moving objects other problems gather with the fore mentioned ones, in particular camera synchronization and motion blur [17].

\section{MONTE CARLO SIMULATION}

The approach proposed by the authors sees the application of Monte Carlo methodology [11], [12] in order to simulate the whole measurement process. This choice is claimed to be the most accurate when estimating uncertainty of complex measurement systems [23]-[25]. Firstly, an image based measurement technique to evaluate is selected. Then it is necessary to select a structure to be monitored and recover a dynamic response model as a set of Laplace domain transfer functions (LTI model). The variability of structural response is represented by sampling modal parameters (mainly natural frequencies and damping ratios) from suitable probability density functions (PDFs). Then it is necessary to sample a structural excitation time history $F(t)$, by means of parametric analytic functions $F_{0}\left(t, p_{1}, p_{2}, \ldots, p_{n}\right)$ having the $p_{n}$ parameters of (1) sampled from appropriate PDFs. It is also possible to add some process noise $N_{p}(t)$.

$F(t)=F_{0}\left(t, p_{1}, p_{2}, \ldots, p_{n}\right)+N_{p}(t)$.

The next step sees the simulation of mechanical response by inputting structural excitation into the LTI model. Consequently, an ideal displacement time history $x_{I D}(t)$ is retrieved. Then, by using a displacement transducer model, it is possible to calculate the measured displacement $x_{T R}(t)$ from $x_{I D}(t)$. At the same time the ideal displacement time history is used to simulate the image formation process. In particular, it is possible to take into account motion blur, optical aberration and lighting changes using a proper image formation model that will be further discussed. Once the images of vibrating target are recovered, it is possible to send them to the processing stage, where the vision based method is used to recover a vision based displacement signal $x_{V I S}(t)$. Furthermore, starting from $F(t)$, the excitation measurement $\tilde{F}(t)$ is calculated from an appropriate transducer model.

Up to this stage of the algorithm, two displacement signals of the structure are available: one coming from usual transducers $x_{T R}(t)$, another from vision system $x_{V I S}(t)$ and the excitation signal $\tilde{F}(t)$. Therefore, it is possible to send them to a transfer function estimation algorithm in order to estimate the modal parameters of interest.

The previous sequence of operations provides a single realization of the value of monitored modal parameters for a given structure in case of traditional transducers and vision based measurement systems. By running those operations sequentially for a high number of times, we get the Monte Carlo loop described in Figure 1.

\subsection{Simulating image formation}

The process of image formation is quite complex and it should be simulated by providing some simplifying assumptions. In particular, the rigid motion of the structure is simulated by phase shifting the Fourier Transform of the image of the target in equilibrium position [26]. Simultaneously motion blur is generated using the averaging algorithm [27]. This transformation is known as the EPSA (Exact Phase Shift and Averaging) algorithm. 


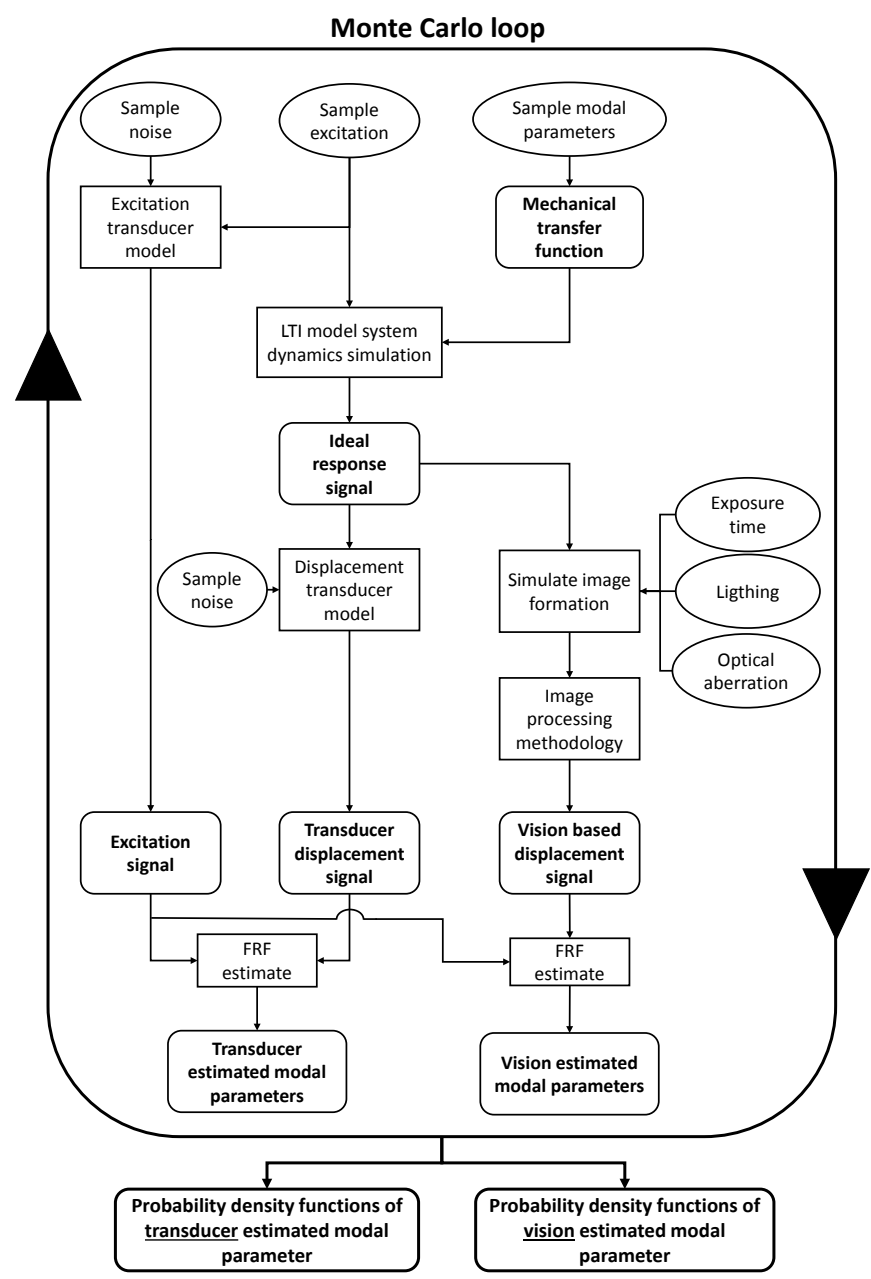

Figure 1. Monte Carlo loop description: single realization of the monitored structure.

Then it is possible to add contrast stretch or amplification with operations on the image histogram. Optical aberration may be modelled by resampling the image with a quadratic or cubic radial aberration model. Other contributes (optical blur, noise..) can be modelled with appropriate image filters.

\section{NUMERICAL STUDY: ANALYZING THE EFFECTS OF MOTION BLUR ON FRF ESTIMATION}

A simple case study is proposed in order to evaluate the effects of motion blur on Frequency Response Function (FRF) estimation and validate the probabilistic architecture here proposed [28]. For the purpose of this paper, a linear time independent (LTI) model of the flexural behaviour of a stadium grandstand is simulated. The parameters were extracted from [7] to provide a realistic example. The model represents the vertical displacement response of a platform tip for a vertical force for the first three natural frequencies. It can be expressed as a parallel of three mono-modal transfer functions in the Laplace domain $G_{i}(s)$ in (2).

$\left\{\begin{aligned} & G_{i}(s)= \frac{1}{\widetilde{m}_{i} s^{2}+2 \widetilde{m}_{i} h_{i} \omega_{i} s+\widetilde{m}_{i} \omega_{i}^{2}} . \\ & \omega_{i}=2 \pi f_{i}\end{aligned}\right.$

Modal masses $\tilde{m}_{i}$ are fixed, while the natural frequencies $f_{i}$ and damping ratios $h_{i}$ are random variables. The stochastic nature of $f_{i}$ is represented by normal PDFs, while the variability of damping ratios is expressed by a random uniform variable.
The PDFs for each variable are listed in Table 1. The assumptions on probability distributions of modal parameters come from the analysis of a set of data of the authors' personal experience as well as from a review of documented experimental cases found in scientific literature [29], [30].

The modal testing system simulated in this paper is a simple decay test. The excitation force is transduced by a simulated load cell having a signal-to-noise ratio (SNR) equal to 5 . The displacement $x_{T R}(t)$ is transduced by a simulated TOF laser having a SNR equal to 10 . In this case the authors considered to simulate correctly calibrated instruments, for which the transducers noise can be modeled as zero-mean Gaussian. This assumption accords with the type A uncertainty model of a calibrated transducer [10]. Structure excitation is modeled with (3), where $\alpha$ is a random uniform variable with mean 5.6 and standard deviation 0.0115 . This choice provides a smoothed impulse, so (3) reproduces the impulse smoothing behavior of hydraulic actuators [31]. Excitation peak $F_{0}$ is $1000 \pm 50 \mathrm{~N}$ uniformly distributed. Process noise $N_{p}(t)$ for a mechanical actuating device can be modelled as zero mean Gaussian [32] with standard deviation equal to $100 \mathrm{~N}$. The standard deviation of process noise accords with the actually available actuation accuracy for 1-10 kN size hydraulic force actuators [33].

$F\left(t-t_{0}\right)=\frac{F_{0}}{e \cdot \alpha} \cdot\left(t-t_{0}\right) e^{-\alpha \cdot\left(t-t_{0}\right)}+N_{p}(t)$.

For the purpose of this paper, all contributions to uncertainty related to instrument calibration are neglected. Both the signals are sampled at $50 \mathrm{~Hz}$ and processed using the wellknown Welch periodogram method in order to estimate the structural FRF. The estimated FRF is calculated with a frequency resolution of $0.01 \mathrm{~Hz}$. In order to avoid windowing problems, the excitation starts at $t_{0}=33 \mathrm{~s}$.

In this case study, the modal parameters monitored are the first two resonant frequencies $f_{1}, f_{2}$ and their relative resonant amplitudes $a_{1}, a_{2}$ (which are the amplitude of estimated FRF at the estimated resonant frequencies). Resonant frequencies are identified by peak picking the absolute value of the imaginary part of FRF. Since the structure's displacement is monitored at the same time by a classic transducer and by a vision technique, it is possible to compare the estimation of the fore mentioned modal parameters provided by the vision system with the reference one provided by the TOF laser.

\subsection{Imaging technique and investigation path}

For what concerns the case study here presented, vibrations are monitored with a simple $2 \mathrm{D}$ blob detection method. This method has been chosen due to its simplicity and straightforward implementation inside a Monte Carlo loop. Nonetheless Blob Analysis is a widespread and reliable methodology. For sure it is possible to simulate more modern and sophisticated methods (i.e. pattern matching, digital image correlation), however the effects of motion blur are expected to behave similarly: motion blur is able to degrade all the

Table 1. PDFs of structural response variability.

\begin{tabular}{cccc}
\hline Variable & PDF & Mean & Std. dev. \\
\hline$f_{1}$ & Normal & 2.0 & 0.1 \\
$h_{1}$ & Uniform & 0.06 & 0.0014 \\
$f_{2}$ & Normal & 2.5 & 0.05 \\
$h_{2}$ & Uniform & 0.03 & 0.0003 \\
$f_{3}$ & Normal & 3.16 & 0.2 \\
$h_{3}$ & Uniform & 0.15 & 0.0029 \\
\hline
\end{tabular}


geometrical features nested in an image, so that all the existing segmentation methods suffer from the presence of motion blur [34], [35]. The simulated blob consists in a bright circle on a black background. The luminance profile of the blob is flat with edges smoothed by a Gaussian blur. Blob detection is carried out by a fixed threshold, then the centroid of the bright region is computed.

For each sample of structure displacement, the reference blob image is proportionally translated. For the purpose of this paper, the amount of translation is directly proportional to $x_{I D}(t)$ by means of a simple scaling factor equal to $20 \frac{\mathrm{px}}{\mathrm{mm}}$. Up to now, an image of the tracked blob without motion blur is available for each sample of the actual displacement of structure $x_{I D}(t)$ (in fact the frames are virtually shot with infinitesimal exposure time as in Figure 2a).

As said previously, the aim of this work is to investigate the effect of motion blur. This is done by averaging the rigidly translated blob images up to a specified exposure time [27]. In this way the actual physics of motion blur formation is simulated: during exposure time the blob travels through the recorded scene. The final result of this procedure is shown in Figure $2 \mathrm{~b}$ where it is possible to see how motion blur smears shapes by convoluting marker motion with the acquisition window.

In this case study nine levels of exposure times are tested: $40,60,80,100,120,140,160$ and $180 \mathrm{~ms}$. The specified path corresponds to exposure to period ratios E2PR [36] spanning from 0.04 up to 0.4 . The testing path here described investigates motion blur starting from the typical condition of a performing experimental setup (where E2PR spans in the range 0.01-0.08 [36]) ending to high motion blur situations (where exposure is about $40 \%$ of the vibration period). $E 2 P R$ has been demonstrated to be one of the most relevant parameters to estimate the effect of motion blur on uncertainty of vibration imaging. In fact, when the exposure to period ratio is close to zero, images are recorded in quasi-static, conversely high values will generate visible motion blur. Eventually $E 2 P R$ has been proven a valuable index to evaluate the performance of a vision rig applied in vibration monitoring tasks [36]. Higher exposure configurations have not been tested due to the obvious impossibility to measure due to aliasing.

\subsection{Monte Carlo loop and probabilistic analysis}

For each value of exposure time, the Monte Carlo analysis saw the realization of 1000 structure excitation and modal parameter estimations. Each realization saw the simulation of $100 \mathrm{~s}$ of system dynamics, for a total amount of $27 \mathrm{~h}, 46 \mathrm{~min}$ and $40 \mathrm{~s}$ of simulated response for each value of exposure time.

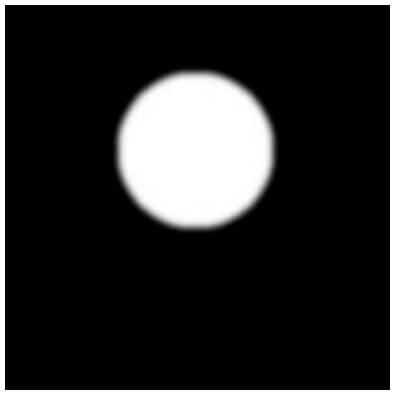

a)

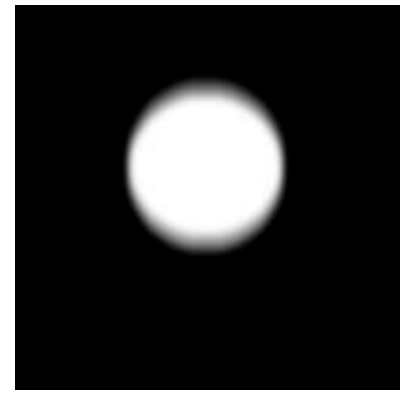

b)
Figure 2. Tracked blob at peak of excitation. On the left the ideal image of marker, on the right motion blur simulation (exposure time $140 \mathrm{~ms}$ ).
At the end of the simulation, for each value of frequency it was possible to retrieve 1000 realizations of FRF for both vision and transducer, consequently 1000 realizations of modal parameters. From this set of values, it was possible to compute the posterior PDFs and their relative cumulative density functions (CDFs). Then it was possible to estimate, for each frequency value, the mean value of FRF and $90 \%$ confidence bounds from the posterior CDFs.

To sum up, as a final output, for each value of exposure time tested in this case study and for both vision and transducer, it was possible to retrieve: estimated FRF with mean and posterior confidence bounds, PDFs of estimated resonant frequencies and PDFs of estimated resonant amplitudes. Eventually, uncertainty is not treated by postulating a particular uncertainty model, but it is computed "as is" from the simulation data.

\subsection{Discussion of simulation results: motion blur as a particular kind of filter}

Once the system has been simulated for all the values of exposure time, it is possible to analyse data of FRF estimation and modal parameters in order to draw some conclusions. In Figure 3 it is possible to see the overall effects on FRF estimation. The vision-estimated response appears smaller in magnitude and more damped in average. The confidence bounds are similar in shape with respect to the transducerbased estimation.

By looking at the diagrams of Figure 4, it is possible to state that using a vision system instead of a transducer has no effect on the identification of natural frequencies, since both the measurement systems display identical identification statistics. Or, at least, no effect is recognizable until exposure time is lower than the frequency aliasing threshold.

Conversely, the process of resonant amplitude identification is affected by the use of vision techniques. The statistical analysis highlights that the presence of a finite exposure time leads to an underestimation of $a_{1}, a_{2}$. As a general trend, the higher the exposure time, the lower is the identified resonant amplitude.

In Figure 5, the statistics of the resonant amplitude identification process are plotted for each value of exposure time. As a result, a graph displaying the standard deviation of

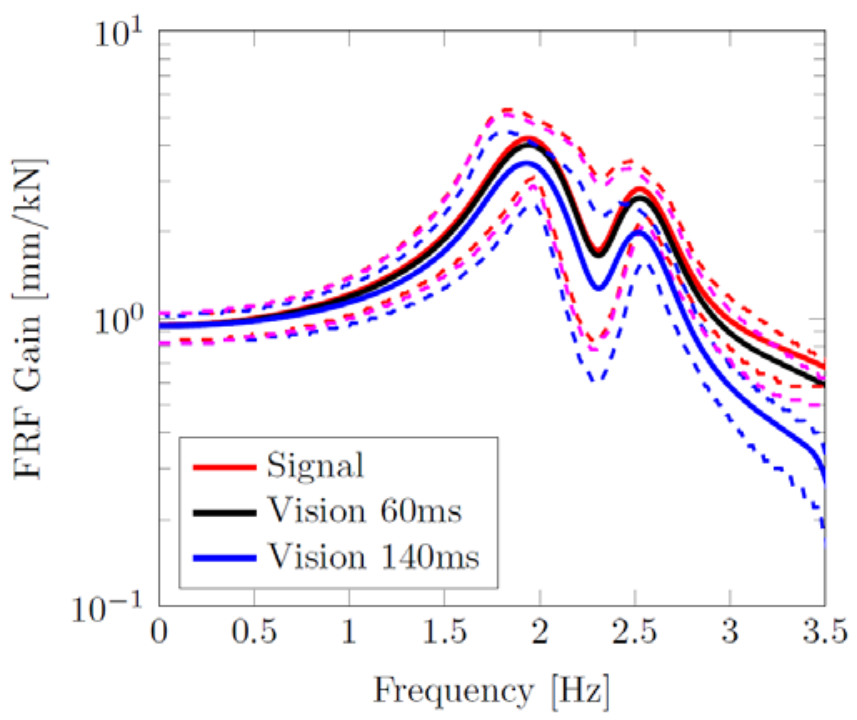

Figure 3. FRF gain diagram for both monitoring techniques with $90 \%$ confidence bounds (exposure time 140 and $60 \mathrm{~ms}$ ). 

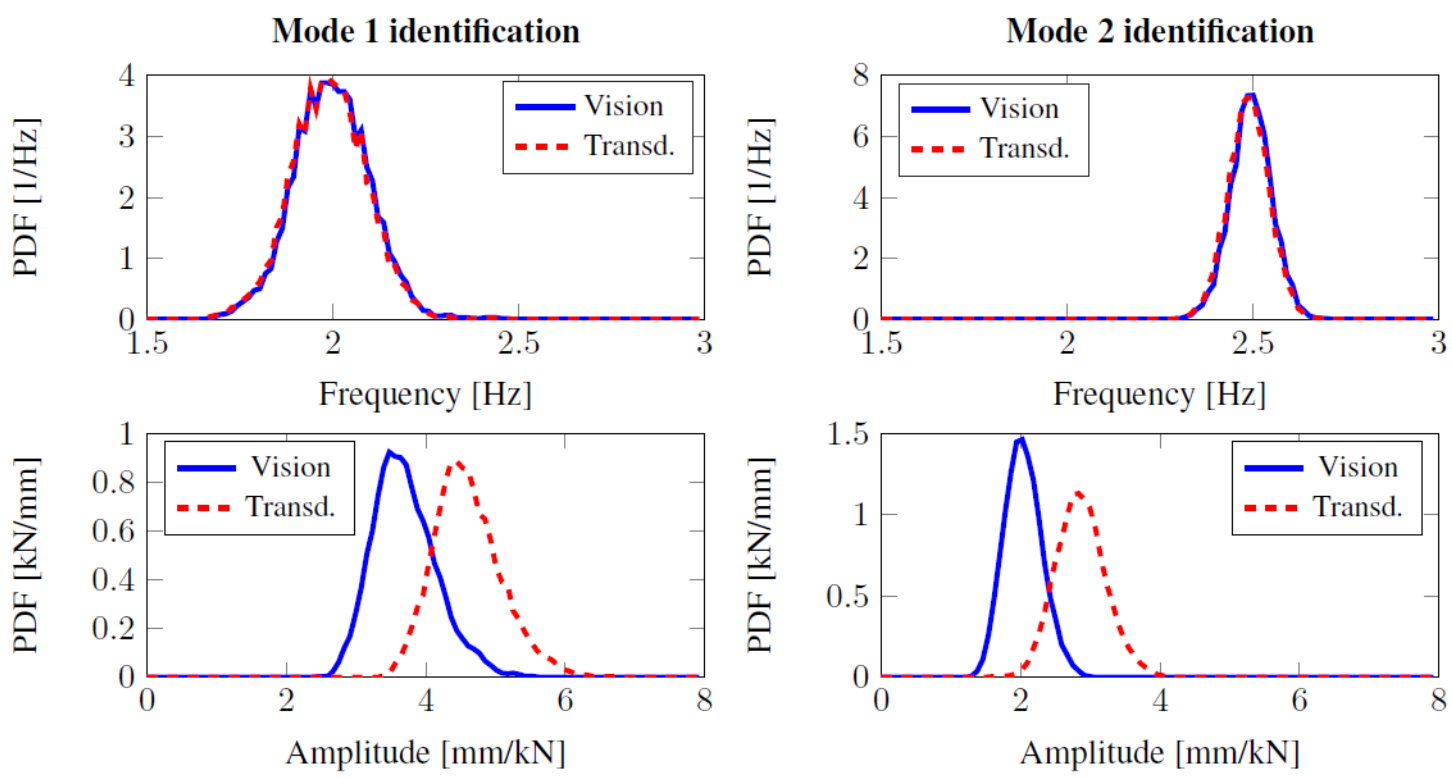

Figure 4. Posterior PDFs of modal parameters identification for exposure time equal to $140 \mathrm{~ms}$.

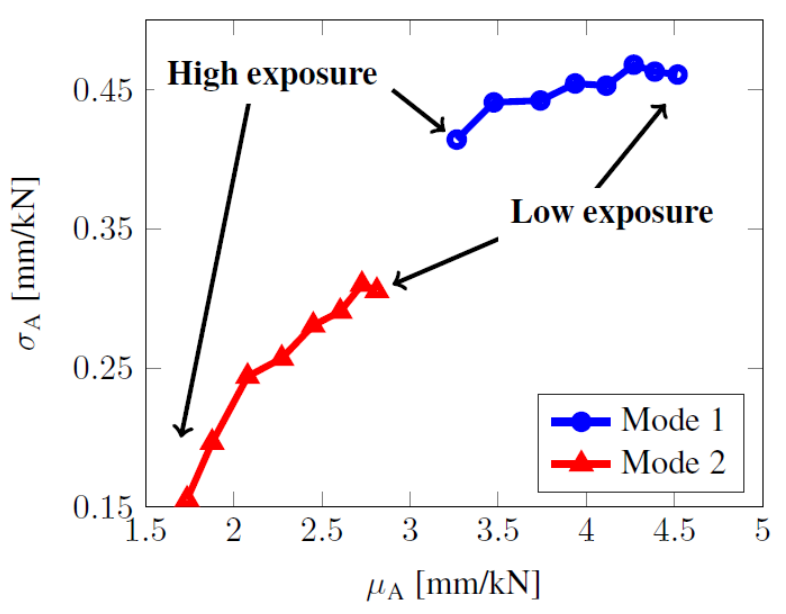

Figure 5. Statistics of identification of resonant amplitudes for the vision based system as motion blur increases.

identified amplitude $\sigma_{A}$ as a function of the mean value of identified amplitude $\mu_{A}$ is shown. As exposure time and motion blur increase, not only the mean value of resonant amplitude decreases, but also the standard deviation of resonant amplitudes decreases. This result is itself interesting:

- as a first effect, motion blur acts as a moving average filter. This is an expected phenomenon and it explains why the mean value of resonant amplitude is shifted to a lower value with respect to a classic transducer. As any mobile average filter [37] it decreases the useful bandwidth of transfer function estimation with a sinc kernel. In particular motion blur produces a clipping of the vibration amplitude at the peak of the sinusoidal motion, as demonstrated in [27];

- a second effect of motion blur is to decrease the standard deviation of resonant amplitude estimation. This phenomenon might lead to the incorrect and risky conclusion that the uncertainty on amplitude estimation is reduced. A physical interpretation of this phenomenon is not found in literature. However, in authors' opinion, the phenomenon of clipping at the inversion point restrains the space of observable states of the system. In other words, as motion blur increases, it is possible to have a set of system configurations that can produce the same measured transfer function due to the filtering effect of the convolution phenomenon.

To sum up, using vision based measurement techniques to monitor vibrations may produce a relevant bias in the evaluation of physical properties of mechanical systems. In the case here discussed, the apparent stiffness is lower and less variable with respect to the ideal case. This phenomenon is triggered by motion blur: the higher is $E 2 P R$, the lower the estimated resonant amplitude. For the system discussed in this case study, the $E 2 P R$ limit for accurate FRF estimation is about 0.12 . Higher values of exposure will produce estimation errors higher than $10 \%$ of the nominal values.

In Figure 6 the width of FRF uncertainty band is plotted as a function of frequency. In this case the presence of a fictitious

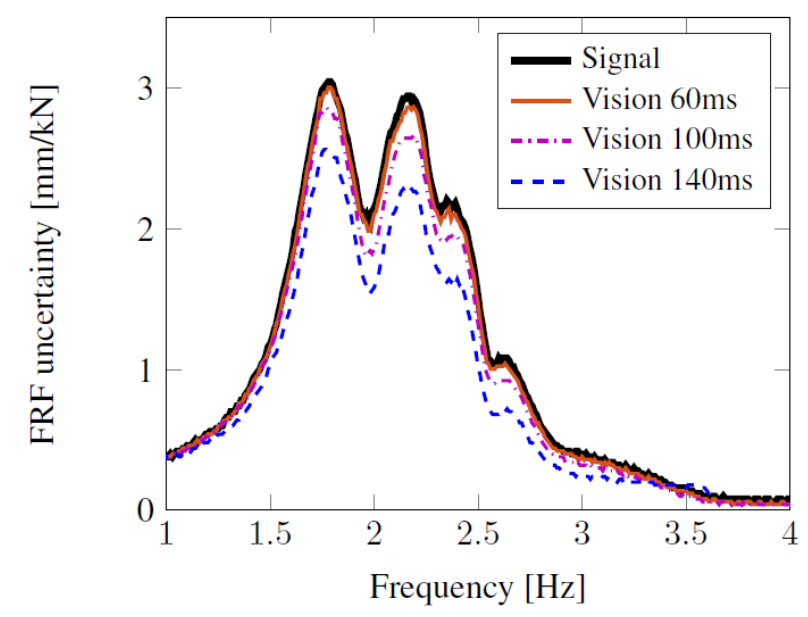

Figure 6. Uncertainty of FRF amplitude estimation (with $90 \%$ level of confidence) for different values of exposure time. 
decrease of uncertainty is detectable in all the process of FRF estimation. As a consequence, the estimation of modal parameters in a single run appears more reliable. This behavior is potentially dangerous in the field of model based SHM, where the value of SNR and the stability of modal parameters are often used to judge the accordance of the experimental behaviour of a structure with the one predicted by a model.

\subsection{Evaluating the uncertainty contributions of the measurement method}

One last analysis available within the proposed uncertainty estimation framework is the evaluation of the uncertainty generated by the modal analysis method. This is done by erasing the uncertainties of the structural response so that the standard deviation of the parameters listed in Table 1 is identically equal to 0 .

In this way, an ideal noise-free transducer should provide a perfect identification of modal parameters, which should be estimated without uncertainty. Conversely all transducer models used in the Monte Carlo simulation take into account disturbances such as electrical noise for analog sensors and motion blur for cameras. Nonetheless, the structural excitation model takes into account process noise. As a consequence, the measurement method itself introduces uncertainty in the estimation of modal parameters, even in the case of an ideal structure [7]. To sum up, it is possible to provide an estimation of measurement uncertainty for the generic modal parameter.

In Table 2 the results of modal parameter estimation at various levels of exposure are listed. Table 2 reports for the first two modes the following quantities: the value of exposure time $t_{s h}$, the mean value of resonant amplitude $\mu_{A}$, the standard deviation of resonant amplitude $\sigma_{A}$, the mean value of resonant frequency $\mu_{F}$ and the standard deviation of resonant frequency $\sigma_{F}$. For what concerns the method used in this case study, the results confirm that motion blur is able to alter the output of modal analysis by introducing measurement bias and changing the apparent measurement uncertainty on FRF amplitude estimation. A further representation of this phenomenon can be found in Figure 7 where the plot of $\sigma_{A}$ as a function of $\mu_{A}$ is displayed.

For what concerns the estimation of natural frequencies, it is possible to state that the vision based methodology here discussed is not sensitive to motion blur. In fact, for both the modes, $\mu_{F}$ and $\sigma_{F}$ are almost constant in value as exposure changes. Conversely it is possible to see a clear trend for $\mu_{A}$ and $\sigma_{A}$, since both monotonically decrease in value as motion blur increases. At low level of exposure, the decreasing slope is quite slow, conversely at high level of exposure the decreasing slope

Table 2. Uncertainty of the vision based modal parameter estimation method for a set of exposure time values.

\begin{tabular}{|c|c|c|c|c|c|c|c|c|}
\hline & \multicolumn{4}{|c|}{ Mode 1} & \multicolumn{4}{|c|}{ Mode 2} \\
\hline \multirow{2}{*}{$\frac{[\mathrm{ms}]}{t_{s h}}$} & \multicolumn{2}{|c|}{ [mm kN-1] } & \multicolumn{2}{|c|}{$[\mathrm{Hz}]$} & \multicolumn{2}{|c|}{ [mm kN-1] } & \multicolumn{2}{|c|}{$[\mathrm{Hz}]$} \\
\hline & $\mu_{A}$ & $\sigma_{A}$ & $\mu_{F}$ & $\sigma_{F}$ & $\mu_{A}$ & $\sigma_{A}$ & $\mu_{F}$ & $\sigma_{F}$ \\
\hline 40 & 4.44 & 0.32 & 2.01 & 0.01 & 2.78 & 0.24 & 2.50 & 0.01 \\
\hline 60 & 4.36 & 0.31 & 2.01 & 0.01 & 2.69 & 0.22 & 2.50 & 0.01 \\
\hline 80 & 4.23 & 0.31 & 2.00 & 0.01 & 2.57 & 0.21 & 2.50 & 0.01 \\
\hline 100 & 4.07 & 0.28 & 2.01 & 0.01 & 2.42 & 0.20 & 2.51 & 0.01 \\
\hline 120 & 3.90 & 0.27 & 2.00 & 0.01 & 2.25 & 0.18 & 2.50 & 0.01 \\
\hline 140 & 3.67 & 0.25 & 2.01 & 0.01 & 2.05 & 0.16 & 2.50 & 0.01 \\
\hline 160 & 3.45 & 0.24 & 2.01 & 0.01 & 1.83 & 0.14 & 2.51 & 0.01 \\
\hline 180 & 3.21 & 0.22 & 2.00 & 0.01 & 1.67 & 0.10 & 2.50 & 0.01 \\
\hline
\end{tabular}

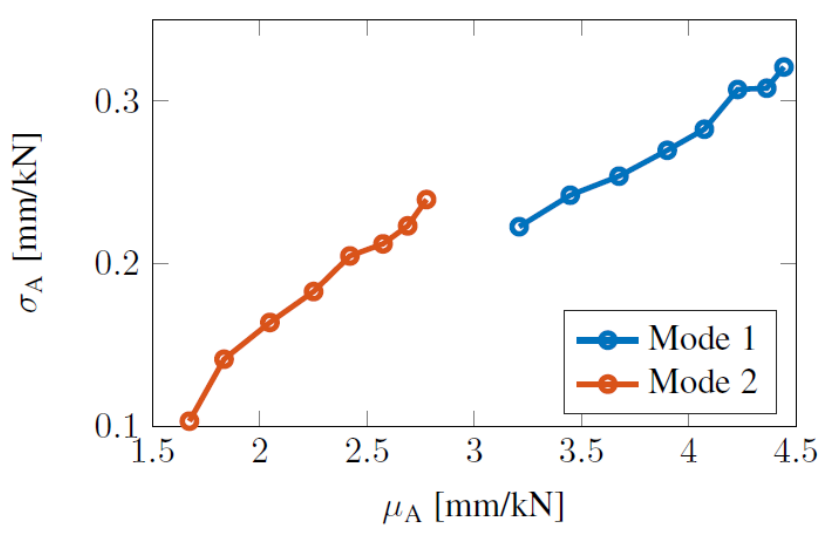

Figure 7. Trends of apparent uncertainty reduction due to motion blur for the vision method analysed.

is steep, resulting in a non-linear behaviour.

The results also confirm the disguising behaviour of motion blur. At low $E 2 P R$ values the presence of this contribution is indeed negligible for a good estimation of modal parameters. However, the $E 2 P R$ depends not only on the exposure time, but also on the period of vibration. Hence each natural frequency is characterized by a different value of E2PR. In conclusion, the estimation of uncertainty in this context is not only related to the method itself, but also to the dynamics of the oscillating system under analysis.

\section{EXPERIMENTAL ACTIVITY}

The probabilistic method proposed previously is claimed to give a glimpse into the critical aspects of the application of vision based measurement techniques for modal analysis purposes. In order to verify the goodness of the probabilistic approach, this paper reports the results of an experimental activity designed for model verification purposes.

\subsection{Design of the experiment}

The aim of the experimental activity is to test the conclusions discussed in the previous part of the paper. To do so, the basic idea is to replicate the scenario simulated in Section 4 with a real mechanical system. More in details, the concept of the experiment is the following:

- pick a mechanical assembly;

- equip the assembly with a vibration exciting device;

- install vibration monitoring transducers on the mechanical assembly;

- select a vision rig in order to perform vision based vibration measurement;

- then, for each value of exposure time, run modal testing several times;

- after that, analyse data and retrieve the statistical distribution of the modal parameters of interest.

For sure, the selection of the total amount of modal tests run for a single value of exposure time is crucial to the statistical significance of the experiment. Nonetheless, time limitations make it impossible to replicate exactly the testing path described in the Monte Carlo simulation presented in Section 4. In fact, in that simulation FRF was estimated with a frequency resolution of $0.01 \mathrm{~Hz}$, which meant about 28 hours of simulated acquisition for each value of exposure time. Considering the fact that 8 levels of exposure time have been tested, the replication of the experiment would require to 
acquire 224 hours of data, coming from the selected mechanical systems. In order to reduce the time needed for the experiment, two solutions have been evaluated: to reduce the number of modal testing for each value of exposure time or to reduce the frequency resolution of FRF estimation. The first one was discarded due to the risk of losing statistical significance. The second one, conversely, has been selected because the quality of FRF estimation wouldn't suffer as long as the natural dynamic of the system is more than 50 times the frequency resolution (this means that the natural frequency of the mechanical assembly must be above $5 \mathrm{~Hz}$ ).

Consequently, the authors chose to run validation on a purposely designed mechanical system, so that modal tests could be run on 10 seconds time histories. In this way it has been possible to run modal testing 1000 times for each value of exposure time. In addition, only for 3 levels of exposure time (2 $\mathrm{ms}, 4 \mathrm{~ms}$ and $6 \mathrm{~ms}$ ), so that the total amount of acquisition time is limited to 8 hours and 20 minutes.

\subsection{Design of the mechanical assembly}

The main goal was to find a simple and reproducible mechanical system, so that all the sources of uncertainties in system behaviour would be under control. Furthermore, the system has to be easily accessible for instrumentation. From what concerns the dynamic characteristic, the first two natural frequencies should be contained in the interval 5-50 Hz: the lower limit is discussed in the previous subsection, while the upper one is chosen in order to avoid aliasing, since the camera's framerate is limited to $120 \mathrm{fps}$.

To keep the assembly as simple as possible, the choice was to mount a cantilever rectangular section beam on an electromagnetic shaker, so that the encastre is actuated vertically with motion $x_{F}(t)$ by the shaker rod (as shown in Figure 8). The beam material is aluminium and its section is 4 $\mathrm{mm}$ thick and $30 \mathrm{~mm}$ wide. The cantilever length is $600 \mathrm{~mm}$, which locates the first natural frequency at $9.1 \mathrm{~Hz}$ and the second one at $57 \mathrm{~Hz}$. This last value is obviously not compatible with the constraints formulated above.

To reduce the value of the second natural frequency, it was decided to put a $0.23 \mathrm{~kg}$ mass on the beam, around the antinode of the second mode shape. In fact, around that point the Lagrangian component of acceleration is much higher for the second mode than for the first one. In order to select the correct position of the mass, a FEM simulation has been run. In Figure 9 it is possible to see that the optimal point was found between 220 and $240 \mathrm{~mm}$ away from the beam encastre, where the frequency of the second mode is minimum.

With this last choice, the FEM predicted values for the natural frequencies are $f_{1} \approx 7.1 \mathrm{~Hz}$ and $f_{2} \approx 32.4 \mathrm{~Hz}$, which let us explore the effects of motion blur at the values of $E 2 P R$ listed in Table 3.

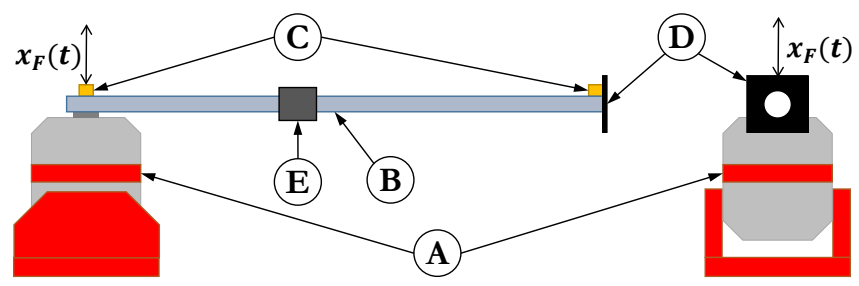

Figure 8. View of the mechanical testing assembly: A) electromagnetic shaker B) cantilever aluminium beam C) accelerometers D) blob analysis target $\mathrm{E}$ ) additional mass.

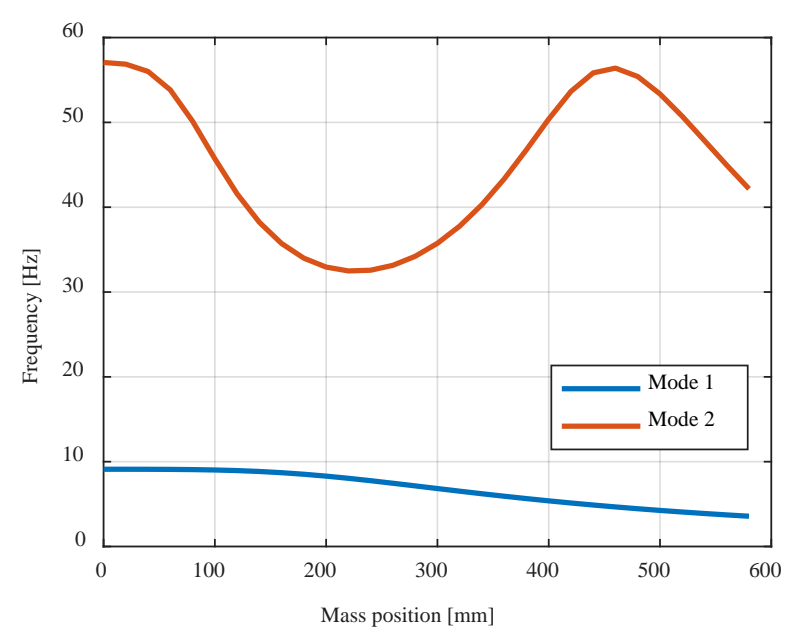

Figure 9. Optimization of the mass position by means of FEM simulation.

Table 3. Values of E2PR investigated during the experimental activity.

\begin{tabular}{ccc}
\hline $\begin{array}{c}\text { Exposure time } \\
{[\mathrm{ms}]}\end{array}$ & Mode 1 E2PR & Mode 2 E2PR \\
\hline 2 & 0.014 & 0.065 \\
4 & 0.028 & 0.129 \\
6 & 0.042 & 0.194 \\
\hline
\end{tabular}

\subsection{Actuating device and instrumentation description}

The selection of the actuating device and the instrumentation is crucial for modal analysis purposes. For what concerns the choice of electromagnetic shakers, it is necessary to ensure the necessary frequency range for the encastre forcing. The choice was then made for a LDS V408 actuator having a useful actuating range of $5-9000 \mathrm{~Hz}$ with an operative maximum sine force peak of $98 \mathrm{~N}$. The maximum peak to peak displacement is $17.6 \mathrm{~mm}$, which is enough to accommodate the minimum desired amplitude of $3 \mathrm{~mm}$ peak to peak. The shaker is powered by a LDS PA500L lock-in amplifier, with a $500 \mathrm{~W}$ power rating. The actuation signal is synthetized with a National Instruments (NI) 9263 analog output module ( $\pm 10 \mathrm{~V}$, 16 bit, $10 \mathrm{kHz}$ bandwidth)

For what concerns the transducer setup, vibrations are then monitored with two PCB 333B30 ICP accelerometers, one positioned on the encastre, one on the beam tip. Their nominal sensitivity is $100 \mathrm{mV} / \mathrm{g}$, with a $\pm 50 \mathrm{~g}$ measurement range and $0.00015 \mathrm{~g}$ RMS resolution. Signals are acquired with NI 9234 analog input module $( \pm 5 \mathrm{~V}, 24 \mathrm{bit}, 51 \mathrm{kS} / \mathrm{s}$, embedded antialiasing filter). Acceleration signals are sampled at $2048 \mathrm{~Hz}$.

Vision measurements are carried out with the help of the well-known blob analysis technique. A painted blob is positioned on the beam tip (Figure 10) and its motion is monitored frontally by a single camera. The camera used in the experiment is an AVT Marlin F131B (focal length of $12 \mathrm{~mm}$ according to ISO 517) mounted on an aluminium tripod. The performances of the electro-optical setup have been evaluated from the 0.10SFR response level according to the ISO 12233 standard [38], showing a sampling efficiency rating $E_{S}$ of $91 \%$. Images have been transferred to the PC using the IEEE 1394 bus and stored in IEEE 12234-compliant TIFF files. After calibration, the value retrieved for the image scaling factor is $0.1266 \mathrm{~mm} / \mathrm{px}$. Images are sampled at $120 \mathrm{fps}$. 


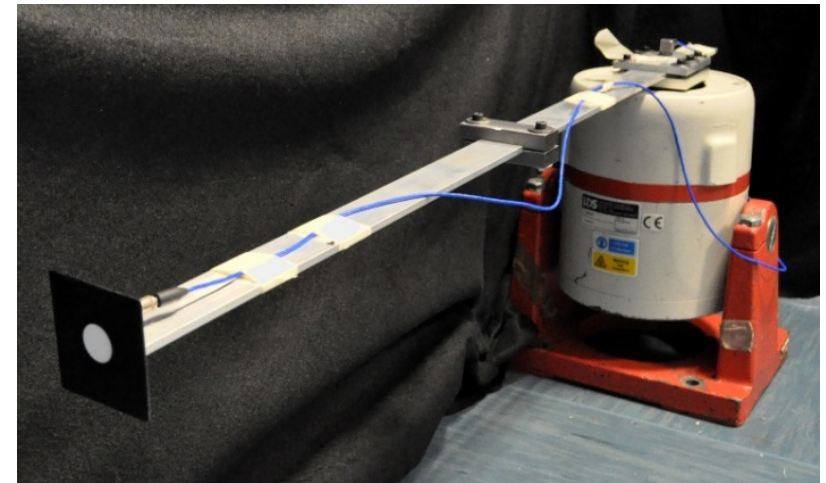

Figure 10. The test-stand as mounted in the laboratory. The white blob is mounted on the beam tip and is frontally recorded by the camera.

\subsection{Data acquisition and processing}

The experimental setup described above has been made with the objective to compare the transducer-based FRF estimation with the vision based one. In this case the FRF input is the acceleration at the encastre, while the output is the acceleration of the beam tip.

When dealing with signals coming from the accelerometers, FRF estimation is done by applying the well-known $\mathrm{H} 1$ estimator [39] on each single modal test acquisition. For what concerns signal windowing, the choice was to use the Hanning window. The reason of this choice is to use a common general purpose window for white noise excitation [40]. When changing the window function, in fact, the shape of FRF around peaks is modified depending on the damping coefficient of each mode [39]. In any case, the peak deformation due to changes in the window function acts equally on the vision estimated FRF and on the accelerometer estimated FRF (and this has been tested on the data by comparing the results of Hanning window against the Flat Top window). Consequently, the conclusions about the effects of motion blur on modal analysis are computed without loss of generality. Then the estimation of the first two resonance frequencies $f_{1}, f_{2}$ is carried out by peak picking the imaginary part of the $\mathrm{H} 1$ estimator. Resonant amplitudes $a_{1}, a_{2}$ are then retrieved by selecting the value of magnitude of $\mathrm{H} 1$ estimator in coincidence of the natural frequencies.

The processing of vision data, conversely, is not straightforward as in the case of accelerometers. The first step sees the segmentation of the image using a simple fixed threshold. Then the center of gravity of the tracked blob is calculated. So, the position of the barycenter with respect to the static position (in px) is available for each frame acquired. Displacement is then calculated by applying the $\mathrm{mm} / \mathrm{px}$ scaling to the previous data. Now we have a signal $x_{\text {vis }}(t)$ that represents the beam tip displacement time history, sampled at $120 \mathrm{~Hz}$. This measure has to be further processed, since the excitation signal is an acceleration sampled at $2048 \mathrm{~Hz}$. The first step is to oversample the vision displacement signal using Fourier interpolation, so that the informative content is not altered. In this way a displacement signal with the same sampling rate of the acceleration signal is available. Now to match displacement with acceleration, the spectrum of $x_{v i s}(t)$ is calculated and then multiplied by $-\omega^{2}$. In the end, it is possible to apply the H1 estimator and estimate the modal parameters also for the vision system.
Another important issue to be solved at the acquisition stage is the synchronization of image acquisition with analog acquisition. This result is achieved by driving camera acquisition and the ADC conversion of the accelerometer signal with two different clocks that share a common time base. In this way the analog acquisition rate and the camera sampling frequency are integer multiples of a common fast time-base, hence it is possible to carry out all digital signal processing steps that require re-sampling and re-phasing in a correct and consistent way.

For each run of modal testing values for natural frequencies and resonant amplitudes are estimated by the accelerometers monitoring system and by the vision monitoring systems. At the end of the test 1000 modal tests are recorded for each value of exposure time, hence 1000 estimation of modal parameters of interest. As a consequence, process statistics (mean, std. deviation, PDFs) are calculated for the $f_{1}, f_{2}, a_{1}, a_{2}$ quantities estimated during the experiment. So that it is possible to further compare the performance of the vision system against the accelerometer-based one.

\subsection{Experimental results analysis}

In this section the results of the experimental activity are presented with a particular focus on investigating how the application of the vision based measurement method may affect the estimation of modal parameters of a structure.

In Figure 11 the FRF estimation over 1000 time histories of $10 \mathrm{~s}$ for the acceleration transducer and vision system for the 3 levels of exposure is presented.

As first comment, it is important to say that, in average, the FRF estimated by the vision system is similar and close to the one of accelerometers. This is the same as predicted by the Monte Carlo simulation. It confirms the validity of approaching vibration monitoring with vision based measurements, since the mechanical behaviour of the structure is resembled correctly in large part.

However, by zooming the peaks of the mechanical FRF, it is possible to see clearly the action of exposure time, since the FRF peaks are lowered as the motion blur increases. The trend is similar to the one predicted by the Monte Carlo simulation in Figure 3 , hence the probabilistic approach was able to predict the rising of this trend. Furthermore, it is possible to see that the peak lowering and flattening phenomenon is more evident for the second mode, where the E2PR is higher. In particular, the pace with the FRF peak goes down with exposure time is faster for the second mode with respect to the first. Once again this feature has been highlighted also in the Monte Carlo case study.

In order to quantify this phenomenon two dimensionless indexes are formulated:

- $\chi_{\mu}$ which represents the ratio between the average resonant amplitude estimated by the vision system and the one estimated by the accelerometers;

- $\chi_{\sigma}$ which represents the ratio between the standard deviation of resonant amplitude estimated by the vision system and the one estimated by the accelerometers.

Those indexes have been selected because they indicate clearly the phenomenon of reduction of both the mean value and the standard deviation of the resonant amplitude with motion blur as described by the simulation in Figure 4. In fact, by looking at Table 4 it is possible to see that both the indexes display a decreasing trend with motion blur. For both the 


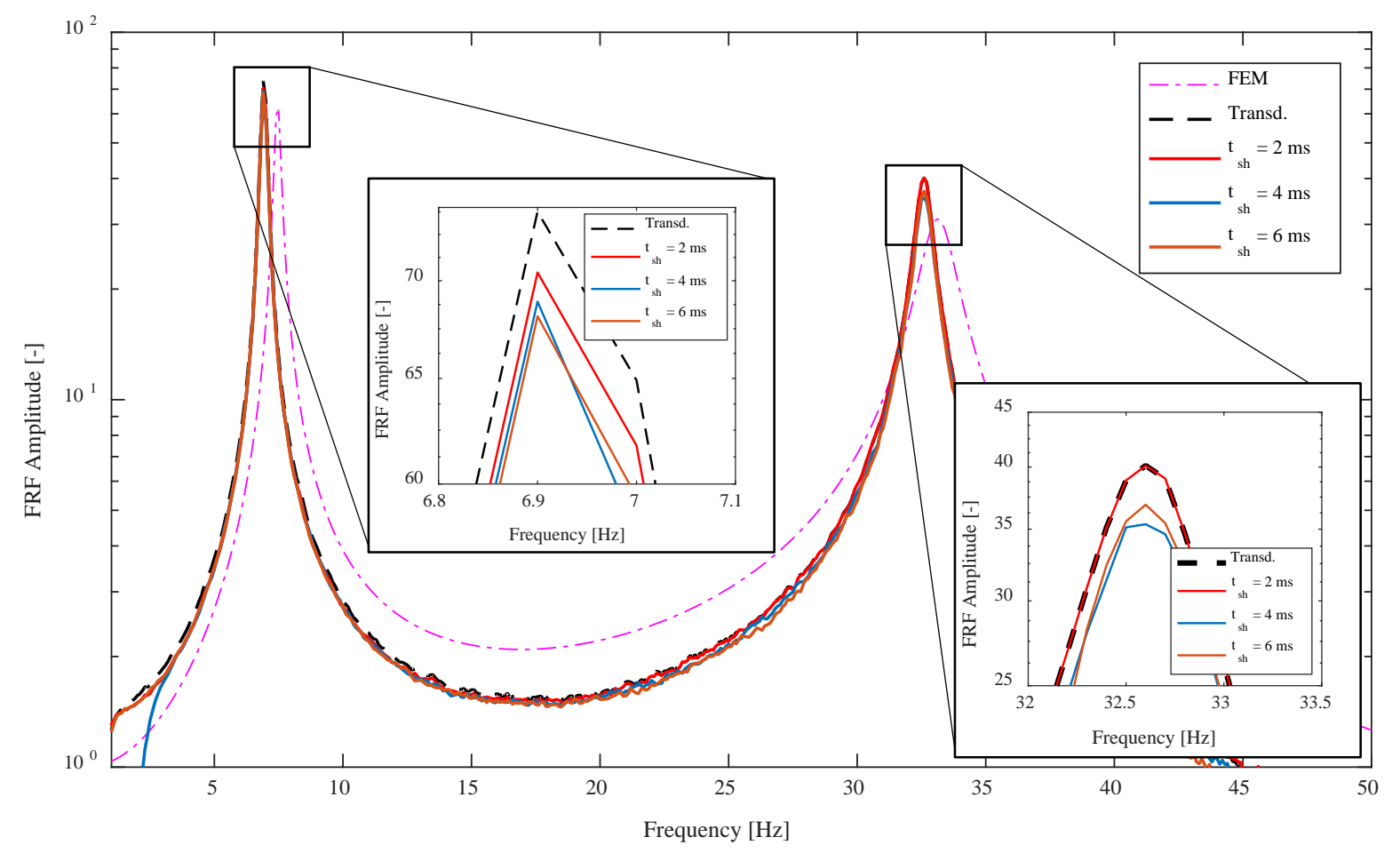

Figure 11. Comparison between FEM simulated FRF, accelerometer based FRF and vision based FRF for different values of exposure time. The curve plotted represents the FRF averaged over 1000 runs.

Table 4. Uncertainty of the vision based modal parameter estimation method for a set of exposure time values.

Mode 1

\begin{tabular}{|c|c|c|c|c|}
\hline$t_{s h}[\mathrm{~ms}]$ & $\chi_{\mu}$ & $\chi_{\sigma}$ & $\chi_{\mu}$ & $\chi_{\sigma}$ \\
\hline 2 & $95.5 \%$ & $94.6 \%$ & $100.0 \%$ & $100.1 \%$ \\
\hline 4 & $95.2 \%$ & $94.4 \%$ & 97.7 \% & $96.4 \%$ \\
\hline 6 & $94.9 \%$ & $93.7 \%$ & $94.3 \%$ & $94.3 \%$ \\
\hline
\end{tabular}

modes their value is below 1 . The blur decreasing trend is more evident for mode 2, as expected by the higher E2PR.

Another feature to highlight is the apparent higher accuracy $\left(\chi_{\mu} \approx 100 \%\right)$ of estimating amplitude of mode 2 with respect to mode 1 at low exposure. This phenomenon is connected with geometrical errors due to the rotation of the beam tip, which, for a given value of displacement, is higher for mode 1 than for mode 2. This is a "static" bias, due to the $2 \mathrm{D}$ Blob Analysis: it happens every time an out-of-plane occurs, since the $3 \mathrm{D}$ motion of a point is projected on the $2 \mathrm{D}$ surface of the image sensor.

For what concerns identification statistics, the analysis process is the same as for the output of the Monte Carlo simulation. In fact, for each value of exposure time 1000 estimations of natural frequencies $f_{1}, f_{2}$ and resonant amplitudes $a_{1}, a_{2}$ are available, hence it is possible to calculate the PDFs for each modal parameter. In Figure 12 the identification statistics for the tests at $t_{s h}=4 \mathrm{~ms}$ are presented as an example. Starting from the natural frequency estimation, data behave according to the predictions of the Monte Carlo study, since, the relative PDFs are quite similar. For mode 1 this is particularly true, while for mode 2 a small shift between the two curves is noticeable. This may seem not according with Figure 11, where the peak position seems the same as for the accelerometer. This happen because the estimation of natural frequencies is made by peak picking the imaginary part of the H1 estimator: so, if we consider small phase errors that may happen during experimental modal analysis, this may not be exactly coincident with the magnitude peak of the $\mathrm{H} 1$ estimator. The uncertainty of estimation is almost similar.

The estimation of resonant amplitudes, conversely, is more complex in experimental cases, compared to what happens in the Monte Carlo simulation. To be more precise, the PDFs are far for the Gaussian hypothesis but in any case, it is possible to see that the probability density peak of the vision system estimation is slightly left shifted. This feature, once again, accords with the Monte Carlo simulation where this phenomenon is more evident because higher values of E2PR have been investigated.

\subsection{Managing the structural excitation during tests}

Vision systems are based on the detection of image motion, hence they are "displacement-based" sensors. In fact, even in the case of image velocimetry techniques, the metrological input is nested in the alteration of the pixel position of the feature detectable in the image. As a consequence, signal-tonoise ratio (SNR) is higher when displacement is high. In straight words, regardless the measurement technique, it is hard to monitor vibrations characterized by very small displacement with vision based instruments.

However, when a structure is excited in a way that a high displacement is generated, nonlinear dynamic phenomena may occur easily. This is an obvious drawback of vision based measurement systems in comparison with modern accelerometers. For instance, if we consider recent structural testing grade accelerometers (such as the ones using in this 

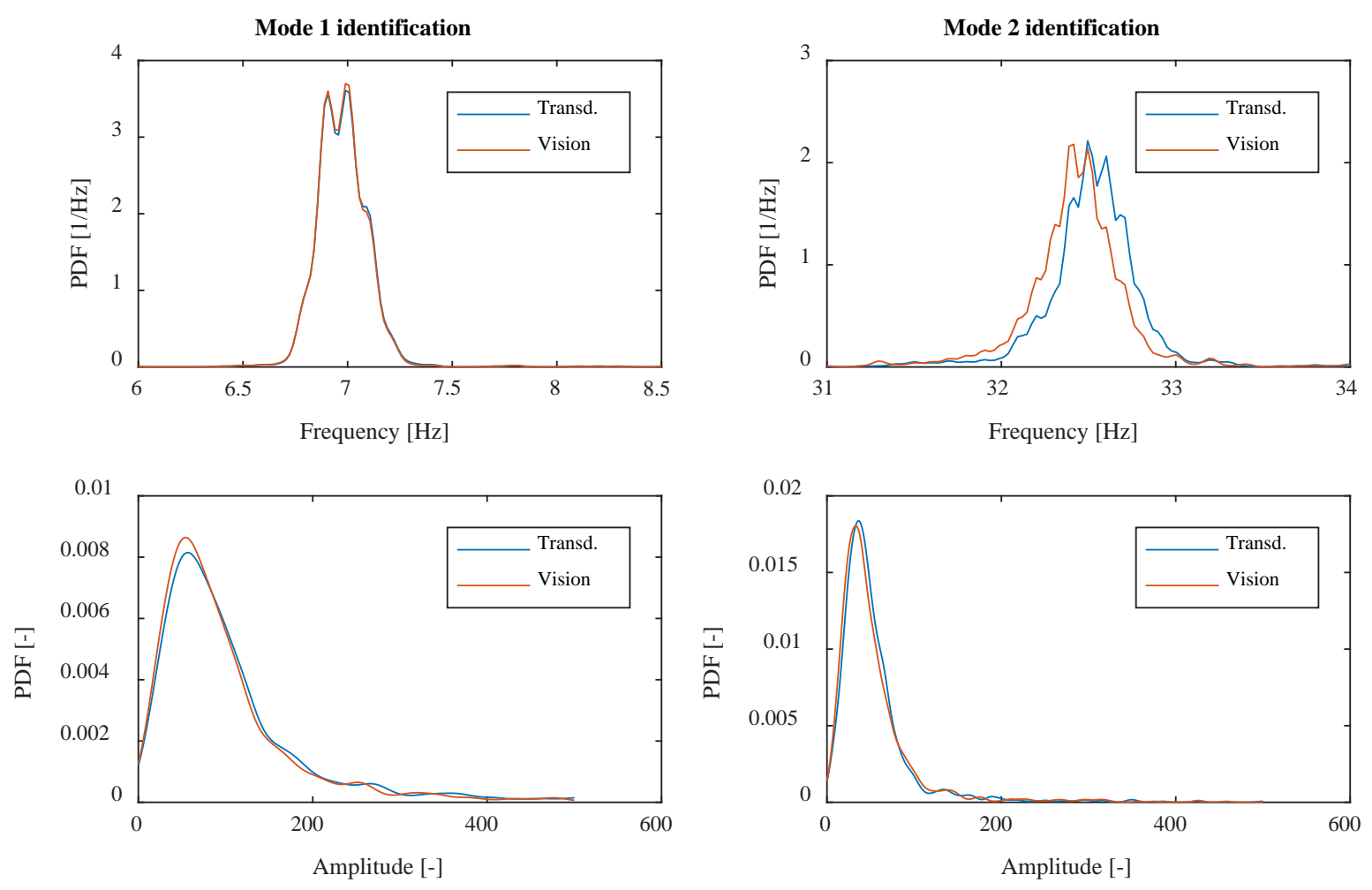

Figure 12. PDFs of experimental modal parameters identification for exposure time equal to $4 \mathrm{~ms}$.

paper) it is common to have a measurement resolution in the $10^{-4} \mathrm{~g}$ RMS region or below. As a consequence, modal tests can be run efficiently even with $10^{-2} \mathrm{~g}$ RMS excitation, provided that a high quality DAC system is used. In practice, it means that accelerometer based modal analysis can easily be accomplished in the full linear dynamic region (small displacements).

In principle, when dealing with linear dynamics, modal tests can be run with any kind of excitation (as long as the excitation spectrum covers all the frequency band of interest and the structure motion develops in the linear region) so that, regardless the kind of excitation, the FRF estimation output should not change. When the dynamic is not linear, conversely, FRF estimation is very sensitive to changes in structural excitations.

During the experimental activity, at first, it was decided to use the same excitation used in the Monte Carlo simulation. That excitation signal is composed by two terms: an impulse and an additive process noise. The impulsive part, in particular, generated a beam tip displacement of more than $6 \mathrm{~mm}$ peak-to peak, which is far away from the small displacement/small rotation region. As a consequence, the statistical distribution reflected the presence of the two terms: as can be seen in Figure 13, the distribution of parameters is not plain. For the mode 2 in particular, it is possible to highlight the presence of a peak when the amplitude values are around 270 .

In order to correct this issue, it was chosen to excite the structure with random uniform band limited white noise. In this way the distribution of modal parameters got back to a monomodal situation. This phenomenon is interesting and it is a clear warning that when operating vision based modal analysis the choice of excitation is crucial to detect correctly the dynamics of the structure.

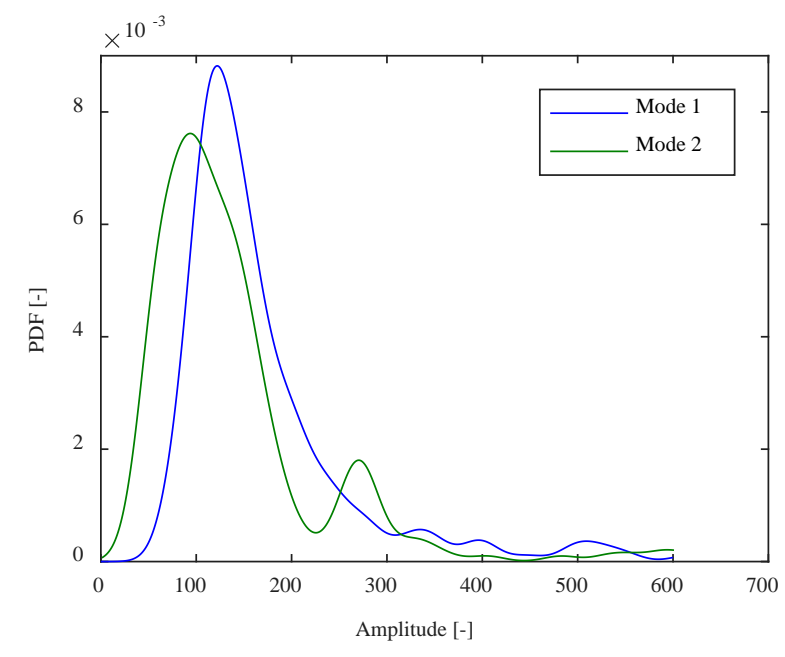

Figure 13. Statistical distribution of resonant amplitudes when exciting the structure with a two component (impulse + white noise) signal.

Another interesting nonlinearity related to excitation in vision based modal analysis is due to motion blur. In fact, motion blur itself is generated by the target moving during exposure. As demonstrated in previous works [27], [35], [36], this phenomenon is driven by the peaks in the instantaneous speed of the measurand. So, if we compare two possible excitation signals, the one that develops higher velocity peaks in the measurand is more prone to produce an under-estimation of resonant amplitude in the H1 estimator (when using vision based measurement system).

To sum up, all the issues discussed in this subsection suggest that the path throughout an extensive development of vision based modal analysis should discuss the problem of finding an 
optimal structural excitation. More in details, the problem is defined by counteracting constraints: displacement should be high so that the SNR is high, but at the same time it should be low to avoid non-linearity. Furthermore, for a correct amplitude estimation, structure excitation should minimize motion blur by controlling the maximum instantaneous speed of the target(s).

\section{CONCLUSIONS}

The presented research work investigated and discussed what are the main limitations of vision based modal analysis in comparison with the classic transducer based application, with particular focus on isolating the main sources of uncertainty in dynamic contexts.

The first part of the research sees the simulation of the measurement process with the help of a Monte Carlo framework. It was found that vision systems may alter the measured behaviour of a structure: in brief, the estimation of resonant amplitude is biased and the bias is higher as the exposure time grows.

Consequently, an experimental activity has been run in order to test the validity of the findings retrieved with the Monte Carlo simulation. The experiments demonstrated that the frequency response measurement bias is present also in real situations, with good statistical significance. Nonetheless, the experiment here proposed suggests that the development of vision techniques in the context of modal analysis should discuss the optimization of structural excitation in order to reduce uncertainty.

\section{REFERENCES}

[1] Zhou, H.F., Dou, H.Y., Qin, L.Z., Chen, Y., Ni, Y.Q., Ko, J.M., "A review of full-scale structural testing of wind turbine blades" (2014), Renewable and Sustainable Energy Reviews, 33, pp. $177-$ 187.

[2] Ellenberg, A., Branco, L., Krick, A., Bartoli, I., Kontsos, A., "Use of unmanned aerial vehicle for quantitative infrastructure evaluation" (2015), Journal of Infrastructure Systems, 21 (3), art. no. 04014054 .

[3] Winkler, J., Hendy, C.R., Waterfall, P., "Improved structural health monitoring strategies for better management of civil infrastructure systems" (2015), Multi-Span Large Bridges Proceedings of the International Conference on Multi-Span Large Bridges, 2015, pp. 855-862.

[4] Desai, N., "Small-strain measurement in bridge connections using the digital image correlation (DIC) technique" (2016), Proceedings of SPIE - The International Society for Optical Engineering, 9805, art. no. 980530.

[5] Busca, G., Cigada, A., Mazzoleni, P., Zappa, E. "Vibration Monitoring of Multiple Bridge Points by Means of a Unique Vision-Based Measuring System" Experimental Mechanics (2014), 54 (2), pp. 255-271.

[6] S. Adhikari, M. Friswell, K. Lonkar, and A. Sarkar, "Experimental case studies for uncertainty quantification in structural dynamics," Probabilistic Engineering Mechanics, vol 24, no. 4, pp. 473 - 492, 2009.

[7] A. Cattaneo, S. Manzoni, and M. Vanali, "Measurement uncertainty in operational modal analysis of a civil structure," in Proceedings of ISMA 2010 - International Conference on Noise and Vibration Engineering, including USD 2010, pp. 5103-5115, 2010.

[8] M. Shinozuka, "Structural response variability", Journal of Engineering Mechanics, vol. 113, no. 6, pp. 825-842, 1987.

[9] C. Bucher and M. Shinozuka, "Structural response variability ii", Journal of Engineering Mechanics, vol. 114, no. 12, pp. 20352054, 1989.
[10] ISO/IEC GUIDE 98-3:2008 - Guide to the expression of uncertainty in measurement (GUM:1995).

[11] ISO/IEC GUIDE 98-3/Suppl.1:2008 - Propagation of distributions using a Monte Carlo method.

[12] E. Zio, "The Monte Carlo Simulation Method for System Reliability and Risk Analysis", Springer Series in Reliability Engineering, Springer Science \& Business Media, 2012.

[13] Hugh W. Coleman and W. G. Steele. "Engineering application of experimental uncertainty analysis", AIAA Journal, Vol. 33, No. 10 (1995), pp. 1888-1896.

[14] Bayarri, M. J., and J. O. Berger. "The Interplay of Bayesian and Frequentist Analysis", Statistical Science, vol. 19, no. 1, 2004, pp. 58-80.

[15] Guyon, I., Saffari, A., Dror, G., Cawley, G., Model selection: Beyond the bayesian/frequentist divide, (2010) Journal of Machine Learning Research, 11, pp. 61-87.

[16] A. Kardara, C. Bucher, and M. Shinozuka, "Structural response variability iii", Journal of Engineering Mechanics, vol. 115, no. 8, pp. 1726-1747, 1989.

[17] J. Baqersad, P. Poozesh, C. Niezrecki, P. Avitabile, "Photogrammetry and optical methods in structural dynamics A review", Mechanical Systems and Signal Processing, 2016, ISSN 0888-3270.

[18] Haralick, B.M., Lee, CN., Ottenberg, K. et al., "Review and analysis of solutions of the three point perspective pose estimation problem", Int J Comput Vision (1994) 13: 331.

[19] D. Cremers, B. Rosenhahn, Alan L. Yuille, Frank R. Schmidt, "Statistical and Geometrical Approaches to Visual Motion Analysis: International Dagstuhl Seminar, Dagstuhl Castle", July 13-18, 2008, Revised Papers, Springer Berlin Heidelberg, 2009.

[20] O. Faugeras, "Three-Dimensional Computer Vision: A Geometric Viewpoint”, MIT Press, 1993.

[21] R. Hartley, A. Zisserman, Multiple View Geometry in Computer Vision. Cambridge University Press, 2004.

[22] Z. Zhang, "A flexible new technique for camera calibration", IEEE Transactions on Pattern Analysis and Machine Intelligence, vol. 22, no. 11, pp. 1330-1334, Nov 2000.

[23] R. Mullen and R. Muhanna, "Probability bounds for the system response of non-linear structures", in Safety, Reliability, Risk and Life-Cycle Performance of Structures and Infrastructures Proceedings of the 11th International Conference on Structural Safety and Reliability, ICOSSAR 2013, pp. 499-502, 2013.

[24] M. Damgaard, L. Andersen, L. Ibsen, H. Toft, and J. Srensen, “A probabilistic analysis of the dynamic response of monopile foundations: Soil variability and its consequences", Probabilistic Engineering Mechanics, vol. 41, pp. 46-59, 2015.

[25] S. Mukhopadhyay, R. Betti, and H. Lus, "Structural identification and uncertainty quantification in operational modal analysis with minimal instrumentation", in Safety, Reliability, Risk and LifeCycle Performance of Structures and Infrastructures Proceedings of the 11th International Conference on Structural Safety and Reliability, ICOSSAR 2013, pp. 1879-1884, 2013.

[26] P. Reu, "Experimental and numerical methods for exact subpixel shifting", Experimental Mechanics, vol. 51, no. 4, pp. 443-452, 2011.

[27] E. Zappa, A. Matinmanesh, and P. Mazzoleni, "Evaluation and improvement of digital image correlation uncertainty in dynamic conditions", Optics and Lasers in Engineering, vol. 59, pp. 8292, 2014

[28] Q. Huang, P. Gardoni, and S. Hurlebaus, "Assessment of modal parameters considering measurement and modelling errors", Smart Structures and Systems, vol. 15, no. 3, pp. 717-733, 2015.

[29] I. Behmanesh and B. Moaveni, "Accounting for environmental variability, modeling errors, and parameter estimation uncertainties in structural identification", Journal of Sound and Vibration, vol. 374, pp. 92 - 110, 2016.

[30] E. Reynders, K. Maes, G. Lombaert, and G. D. Roeck, "Uncertainty quantification in operational modal analysis with stochastic subspace identification: Validation and applications", 
Mechanical Systems and Signal Processing, vol. 6667, pp. 13 30, 2016.

[31] Darby, A. P., Blakeborough, A. and Williams, M. S. (2001), "Improved control algorithm for real-time substructure testing", Earthquake Engng. Struct. Dyn., 30: 431-448.

[32] M. Compare and E. Zio, "Predictive Maintenance by Risk Sensitive Particle Filtering", in IEEE Transactions on Reliability, vol. 63, no. 1, pp. 134-143, March 2014.

[33] Alleyne A, Liu R., "On the Limitations of Force Tracking Control for Hydraulic Servosystems”, ASME. J. Dyn. Sys., Meas., Control. 1999;121(2):184-190.

[34] Y. Wang, P. Lava, P. Reu, and D. Debruyne, "Theoretical analysis on the measurement errors of local $2 \mathrm{~d}$ dic: Part $\mathrm{i}$ temporal and spatial uncertainty quantification of displacement measurements", Strain, 2015.

[35] A. Lavatelli and E. Zappa, "Modeling Uncertainty for a Vision System Applied to Vibration Measurements", in IEEE
Transactions on Instrumentation and Measurement, vol. 65, no 8, pp. 1818-1826, Aug. 2016.

[36] G. Busca, G. Ghislanzoni, and E. Zappa, "Indexes for performance evaluation of cameras applied to dynamic measurements", Measurement: Journal of the International Measurement Confederation, vol. 51, no. 1, pp. 182-196, 2014.

[37] G. D'Antona and A. Ferrero, "Digital Signal Processing for Measurement Systems: Theory and Applications", Information Technology: Transmission, Processing and Storage, Springer US, 2006.

[38] ISO 12233:2014 Photography -- Electronic still picture imaging - Resolution and spatial frequency responses.

[39] D.J. Ewins, "Modal testing: theory, practice, and application", $2^{\text {nd }}$ edition, Research Studies Press, 2000

[40] Gade, S. and Herlufsen, H., "Use of Weighting Functions in DFT/FFT analysis", Brüel \& Kjær Technical Reviews nos. 3 \& 4, 1987. 